\title{
Eigen Values of Interval Matrix
}

\author{
Abhirup Sit \\ Department of Mathematics, IIT Kharagpur
}

In this paper, properties of interval matrix are studied. Some theoretical results on the regularity and the singularity of an interval matrix are explored and verified through several examples. Eigen value of the interval matrix using singularity property is studied. Matlab code is developed to determine eigen values of an interval matrix.

Keywords : Interval Matrix, Regular Interval Matrix, Singular Interval Matrix, Eigen Value of an Interval Matrix.

\section{Introduction}

Interval Analysis is a tool in numerical computing where the rules for the arithmetic of intervals are explicitly stated and applied to what is called today interval arithmetic evaluation of rational expressions. Moore [3] and P.S. Dwyer [1] have discussed matrix computations using interval arithmetic in their book in 1951. The Japanese scientist Teruo Sunaga's [2] paper was one of the most important paper for the development of interval arithmetic. Jiri Rohn's paper like 5, 8, 44 etc are important in this field.

In this chapter we have discussed some priliminary on interval matrix operations.

\section{$1.1 \quad$ Notations}

$\mathrm{X}=$ interval

$\underline{X}$ and $\bar{X}=$ left endpoint and right endpoint of an interval respectively

$\mathrm{A}^{I}=$ Interval matrix

$\mathrm{A}_{\mathrm{c}}=$ Centre of the interval matrix

$\Delta=$ Spread of the interval matrix

$\mathrm{P}$ matrix $=$ Whose principal minors are greater than 0

$\Delta_{1}$ and $\Delta_{2}=$ The first and the second principal minor respectively

$\mathrm{T}_{\mathrm{y}}=$ if $\mathrm{y} \in \mathrm{R}^{n}$ then $\mathrm{T}_{\mathrm{y}}=\operatorname{diag}\left(\mathrm{y}_{1}, \mathrm{y}_{2} \ldots . \mathrm{y}_{\mathrm{n}}\right)$

$\mathrm{Y}=\mathrm{y} ;|y|=\mathrm{e}, \mathrm{y} \in \mathrm{R}^{n}$

$\mathrm{A}_{\mathrm{yz}}=\mathrm{A}_{\mathrm{c}}-\mathrm{T}_{\mathrm{y}} \Delta \mathrm{T}_{\mathrm{z}}$

$\mathrm{A}_{\mathrm{ye}}=\mathrm{A}_{\mathrm{c}}-\mathrm{T}_{\mathrm{y}} \Delta$

$\mathrm{A}_{\mathrm{yf}}=\mathrm{A}_{\mathrm{c}}+\mathrm{T}_{\mathrm{y}} \Delta$

$\mathrm{D}_{\mathrm{y}}=\mathrm{A}_{\mathrm{c}}{ }^{-1} \mathrm{~T}_{\mathrm{y}} \Delta$

$\mathrm{B}=\mathrm{D}_{\mathrm{y}}|B|+\mathrm{A}_{\mathrm{c}}{ }^{-1}$

$\rho_{\mathrm{o}}(\mathrm{A})=$ Eigenvalues of $\mathrm{A}$

$\mathrm{D}_{\mathrm{yz}}=\mathrm{A}_{\mathrm{c}}{ }^{-1} \mathrm{~T}_{\mathrm{y}} \Delta \mathrm{T}_{\mathrm{z}}$

\subsection{Arithmetic Operations on the Set of Intervals}

We will the denote intervals and their endpoints by capital letters. The left and right endpoints of an interval $\mathrm{X}$ will be denoted by $\underline{X}$ and $\bar{X}$, respectively. Thus, $\mathrm{X}=[\underline{X}, \bar{X}]$

Let " . "denotes any binary operation, $\mathrm{X}$ and $\mathrm{Y}$ be any two intervals.

$\mathrm{X} \cdot \mathrm{Y}=[\min \mathrm{S}, \max \mathrm{S}]$, where $\mathrm{S}=\{\underline{X} \underline{Y}, \underline{X} \bar{Y}$, $\bar{X} \underline{Y}, \bar{X} \bar{Y}\}$

Example 1.1. Let $X=[-1,0]$ and $Y=[1,2]$. Then for $\mathrm{X} * \mathrm{Y}, \mathrm{S}=\{-1,0,-2\}$ and $\mathrm{X} * \mathrm{Y}=[-2,0]$

\subsection{Interval Matrix}

Definition 1.1. An interval matrix $A^{I}$ is a matrix whose each elements are intervals.

A genral form of an interval matrix is

$A^{I}=\left(\begin{array}{cccc}\mathrm{a}_{11}^{I} & \mathrm{a}_{12}^{I} & \ldots & \mathrm{a}_{1 n}^{I} \\ \mathrm{a}_{21}^{I} & \mathrm{a}_{22}^{I} & \ldots & \mathrm{a}_{2 n}^{I} \\ \ldots & \ldots & \ldots & \ldots \\ \mathrm{a}_{m 1}^{I} & \mathrm{a}_{m 2}^{I} & \ldots & \mathrm{a}_{m n}{ }^{I}\end{array}\right)=\left(\mathrm{a}_{i j}^{I}\right)_{1 \leq i \leq m, 1 \leq j \leq n}$ where each $a_{i j}^{I}=\left[\underline{a}_{\mathrm{ij}}, \bar{a}_{\mathrm{ij}}\right]$ (or) $A^{I}=[\underline{A}, \bar{A}]$ for some $\underline{A}, \bar{A}$ satisfying $\underline{\mathrm{A}} \leq \bar{A}$

Example 1.2. Take $\mathrm{m}=\mathrm{n}=2$; Let $A^{I}=$ $\left[\begin{array}{ll}{[1,2]} & {[3,4]} \\ {[5,6]} & {[4,7]}\end{array}\right]: A^{I}=[\underline{A}, \bar{A}]=\{\mathrm{A} ; \underline{\mathrm{A}} \leq A \leq \bar{A}\}$

Here, $\underline{\mathrm{A}}=\left[\begin{array}{ll}1 & 3 \\ 5 & 4\end{array}\right], \bar{A}=\left[\begin{array}{ll}2 & 4 \\ 6 & 7\end{array}\right]$

Centre: $\mathrm{A}_{\mathrm{c}}=\frac{1}{2}[\underline{A}+\bar{A}]=\frac{1}{2}\left[\left[\begin{array}{ll}1 & 3 \\ 5 & 4\end{array}\right]+\left[\begin{array}{ll}2 & 4 \\ 6 & 7\end{array}\right]\right]$ $=\left[\begin{array}{cc}3 / 2 & 7 / 2 \\ 11 / 2 & 11 / 2\end{array}\right]$

Spread: $\Delta=\frac{1}{2}(\underline{A}-\bar{A})=\frac{1}{2}\left[\left[\begin{array}{ll}2 & 4 \\ 6 & 7\end{array}\right]-\left[\begin{array}{ll}1 & 3 \\ 5 & 4\end{array}\right]\right]=$ $\left[\begin{array}{ll}1 / 2 & 1 / 2 \\ 1 / 2 & 3 / 2\end{array}\right]$

Also, any $\mathrm{A}^{I}=\left[\mathrm{A}_{\mathrm{c}}-\Delta, \mathrm{A}_{\mathrm{c}}+\Delta\right]$, it can be easily shown. 


\subsection{Determinant of an Interval Ma- trix}

$\operatorname{det} \mathrm{A}^{I}=\left\{\operatorname{det} \mathrm{A} \mid \mathrm{A} \in \mathrm{A}^{I}\right\}=\left[\min _{\forall A \in A}{ }^{I} \operatorname{det}\right.$ $\left.\mathrm{A}, \max _{\forall A \in A}{ }^{I} \operatorname{det} \mathrm{A}\right]$

Example 1.3. Take, $\mathrm{A}^{I}=\left[\begin{array}{ll}{[1,2]} & {[3,4]} \\ {[5,6]} & {[4,7]}\end{array}\right]$; Let $\mathrm{S}$ $=\left\{\operatorname{det}\left[\begin{array}{ll}a & b \\ c & d\end{array}\right]: 1 \leq \mathrm{a} \leq 2,3 \leq \mathrm{b} \leq 4,5 \leq \mathrm{c}\right.$ $\leq 6,4 \leq \mathrm{d} \leq 7\}$

$=\{$ ad-bc $: 1 \leq \mathrm{a} \leq 2,3 \leq \mathrm{b} \leq 4,5 \leq \mathrm{c}$

$\leq 6,4 \leq \mathrm{d} \leq 7\}$

So, $\operatorname{det} \mathrm{A}^{I}=[\min \mathrm{S}, \max \mathrm{S}]=[-20,-1]$

\section{Regular Interval Matrix}

Definition 2.1. A square interval matrix $A^{I}$ is called regular if each $\mathrm{A} \in \mathrm{A}^{I}$ is nonsingular that is $\operatorname{det} \mathrm{A} \neq 0$.

Example 2.1. $\mathrm{S}=\{$ ad-bc $: 1 \leq \mathrm{a} \leq 2,3 \leq \mathrm{b} \leq$ $4,5 \leq \mathrm{c} \leq 6,4 \leq \mathrm{d} \leq 7\}$, if $0 \notin \mathrm{S}$, then each $\mathrm{A}$ is non singular

$$
\operatorname{det} \mathrm{A}^{I}=\operatorname{det}\left[\begin{array}{ll}
{[1,2]} & {[3,4]} \\
{[5,6]} & {[4,7]}
\end{array}\right]=[-20,-1]
$$

since $0 \notin[-20,-1] ; A^{I}$ will be regular

Theorem 2.1. 6 Let $A^{I}$ be regular, then for each $A, A_{1} \in A^{I}$, both $A^{-1} A_{1}$ and $A_{1}^{-1} A$ are $P$ matrices.

Example 2.2. $\mathrm{A}=\left[\begin{array}{ll}1 & 3 \\ 6 & 4\end{array}\right], \mathrm{A}_{1}=\left[\begin{array}{ll}2 & 3 \\ 5 & 4\end{array}\right]$

$$
\begin{aligned}
& A^{-1}=-\frac{1}{14}\left[\begin{array}{cc}
4 & -3 \\
-6 & 1
\end{array}\right] \\
& A^{-1} \mathrm{~A}_{1}=\left[\begin{array}{cc}
1 / 2 & 0 \\
5 / 4 & 1
\end{array}\right] ; \Delta_{1}=\frac{1}{2}>0,
\end{aligned}
$$

$\Delta_{2}=\frac{1}{2}>0$; hence $A^{-1} \mathrm{~A}_{1}$ is P-matrix

Similarly, for $\mathrm{A}_{1}^{-1} \mathrm{~A}$.

Theorem 2.2. 6] Let $A^{I}$ be an $n X n$ interval matrix. Then the following conditions are mutually equivalent:

(R) $A^{I}$ is regular.

(A1) $A_{y z} x=y, T_{z} x \geq 0$ has a (unique) solution for each $y \in Y$; where, $A_{y z}=A_{c}-T_{y} \Delta T_{z}$, $Y=\left\{y ;|y|=e, y \in R^{n}\right\}$.

(A2) $A_{y e} x_{1}-A_{y f} x_{2}=y, x_{1} \geq 0, x_{2} \geq 0$ has a solution for each $y \in Y$, where $A_{y e}=A_{c}-T_{y} \Delta$, $A_{y f}=A_{c}+T_{y} \Delta$.

(A3) $B=D_{y}|B|+A_{c}{ }^{-1}$ has a unique matrix solution for each $y \in Y$, where $D_{y}=A_{c}^{-1} T_{y} \Delta$.

(B1) $A_{y e}{ }^{-1} A_{y f}$ is a P-Matrix for each $y \in Y$

(B2) $A_{y e}{ }^{-1} A_{y f} x>0, x>0$ has a solution for each $y \in Y$.
(B3) $A_{y e}{ }^{-1} x>0$ and $A_{y f}{ }^{-1} x>0$ has a solution for each $y \in Y$.

(B4) If $A^{I}$ is regular matrix. Then $\left|D_{y} x\right|<x$ has a solution for each $y \in Y$.

(C1) $\left(\operatorname{det} A_{y z}\right)\left(\operatorname{det} A_{y^{\prime} z^{\prime}}\right)>0$ for each $y, y^{\prime}, z, z^{\prime}$ $\in Y$.

(C2) $\left(\operatorname{det} A_{y z}\right)\left(\operatorname{det} A_{y^{\prime} z}\right)>0$ for each $y, y^{\prime}, z \in Y$ such that $y$ and $y^{\prime}$ differ in just one entry

(C3) $\rho_{o}\left(D_{y z}\right)<1$ for each $y, z \in Y$, where $D_{y z}=$ $A_{c}{ }^{-1} T_{y} \Delta T_{z}$

(C4) $\left(A_{c} A_{y z}{ }^{-1}\right)_{i i}>1 / 2$; for each $y, z \in Y$; $i \in 1,2, \ldots, n$.

(C5) $\left(A_{c} A^{-1}\right)_{i i}>1 / 2$ for each $A \in A^{I}, i \in$ $i, 2, \ldots, n$

Example 2.3. Take $\mathrm{n}=2$ then

$\mathrm{Y}=\left\{\left[\begin{array}{l}1 \\ 1\end{array}\right],\left[\begin{array}{c}1 \\ -1\end{array}\right],\left[\begin{array}{c}-1 \\ 1\end{array}\right],\left[\begin{array}{l}-1 \\ -1\end{array}\right]\right\}$

$(\mathbf{R}) \rightarrow($ A1 $)$

$\mathrm{z}=\left[\begin{array}{l}1 \\ 2\end{array}\right], \mathrm{y}=\left[\begin{array}{l}1 \\ 1\end{array}\right] ; \mathrm{T}_{\mathrm{z}}=\left[\begin{array}{ll}1 & 0 \\ 0 & 2\end{array}\right] ; \mathrm{T}_{\mathrm{y}}=\left[\begin{array}{ll}1 & 0 \\ 0 & 1\end{array}\right] ;$

$A_{c}=\left[\begin{array}{cc}3 / 2 & 7 / 2 \\ 11 / 2 & 11 / 2\end{array}\right] ; \Delta=\left[\begin{array}{cc}1 / 2 & 1 / 2 \\ 1 / 2 & 3 / 2\end{array}\right]$

$\mathrm{A}_{\mathrm{yz}}=\left[\begin{array}{cc}3 / 2 & 7 / 2 \\ 11 / 2 & 11 / 2\end{array}\right]-\left[\begin{array}{cc}1 & 0 \\ 0 & 1\end{array}\right] \cdot\left[\begin{array}{cc}1 / 2 & 1 / 2 \\ 1 / 2 & 3 / 2\end{array}\right]$.

$\left[\begin{array}{ll}1 & 0 \\ 0 & 2\end{array}\right]=\left[\begin{array}{ll}1 & 5 / 2 \\ 5 & 5 / 2\end{array}\right]$

$\mathrm{A}_{\mathrm{yz}} \mathrm{x} \quad=\mathrm{y} \quad \Rightarrow\left[\begin{array}{ll}1 & 5 / 2 \\ 5 & 5 / 2\end{array}\right] \quad\left[\begin{array}{l}x_{1} \\ x_{2}\end{array}\right]=\left[\begin{array}{l}1 \\ 1\end{array}\right] ;$ $x=\left[\begin{array}{c}0 \\ 2 / 5\end{array}\right] ; T_{\mathrm{z}} x=\left[\begin{array}{ll}1 & 0 \\ 0 & 2\end{array}\right]\left[\begin{array}{c}0 \\ 2 / 5\end{array}\right]=\left[\begin{array}{c}0 \\ 4 / 5\end{array}\right] \geq 0$

$$
(\mathbf{R}) \rightarrow(\mathbf{A 2})
$$

$\mathrm{y}=\left[\begin{array}{c}1 \\ -1\end{array}\right], \mathrm{Ty}=\left[\begin{array}{cc}1 & 0 \\ 0 & -1\end{array}\right]$

$A_{y e}=A_{c}-T_{y} \Delta=\left[\begin{array}{cc}3 / 2 & 7 / 2 \\ 11 / 2 & 11 / 2\end{array}\right]-\left[\begin{array}{cc}1 & 0 \\ 0 & -1\end{array}\right]\left[\begin{array}{cc}1 / 2 & 1 / 2 \\ 1 / 2 & 3 / 2\end{array}\right]=\left[\begin{array}{cc}1 & 3 \\ 6 & 7\end{array}\right]$

$\mathrm{A}_{\mathrm{yf}}=\mathrm{A}_{\mathrm{c}}+\mathrm{T}_{\mathrm{y}} \Delta=\left[\begin{array}{cc}3 / 2 & 7 / 2 \\ 11 / 2 & 11 / 2\end{array}\right]+\left[\begin{array}{cc}1 & 0 \\ 0 & -1\end{array}\right]\left[\begin{array}{cc}1 / 2 & 1 / 2 \\ 1 / 2 & 3 / 2\end{array}\right]=\left[\begin{array}{ll}2 & 4 \\ 5 & 4\end{array}\right]$ $\mathrm{A}_{\mathrm{ye}} \mathrm{x}_{1}-\mathrm{A}_{\mathrm{yf}} \mathrm{x}_{2}=\mathrm{y} ; \mathrm{x}_{1} \geq 0, \mathrm{x}_{2} \geq 0$

$\left[\begin{array}{ll}1 & 3 \\ 6 & 7\end{array}\right]\left[\begin{array}{l}x^{\prime}{ }_{1} \\ x^{\prime \prime}{ }_{1}\end{array}\right]-\left[\begin{array}{ll}2 & 4 \\ 5 & 4\end{array}\right]\left[\begin{array}{l}x^{\prime}{ }_{2} \\ x^{\prime \prime}{ }_{2}\end{array}\right]=\left[\begin{array}{c}1 \\ -1\end{array}\right] ;$ By solving $\mathrm{x}_{1}=\left[\begin{array}{l}0 \\ 7\end{array}\right]$ and $\mathrm{x}_{2}=\left[\begin{array}{c}10 \\ 0\end{array}\right]$

( R ) $\rightarrow$ ( A3 )

$\mathrm{A}_{\mathrm{c}}=\left[\begin{array}{cc}3 / 2 & 7 / 2 \\ 11 / 2 & 11 / 2\end{array}\right] ; \mathrm{A}_{\mathrm{c}}{ }^{-1}=\left[\begin{array}{cc}-11 / 44 & 7 / 44 \\ 11 / 44 & -3 / 44\end{array}\right]$

take $\mathrm{y}=\left[\begin{array}{c}1 \\ -1\end{array}\right], \mathrm{T}_{\mathrm{y}}=\left[\begin{array}{cc}1 & 0 \\ 0 & -1\end{array}\right] ; \Delta=\left[\begin{array}{cc}1 / 2 & 1 / 2 \\ 1 / 2 & 3 / 2\end{array}\right]$

$\mathrm{D}_{\mathrm{y}}=\mathrm{A}_{\mathrm{c}} \mathrm{C}^{-1} \mathrm{~T}_{\mathrm{y}} \Delta=\left[\begin{array}{cc}-11 / 44 & 7 / 44 \\ 11 / 44 & -3 / 44\end{array}\right]\left[\begin{array}{cc}1 & 0 \\ 0 & -1\end{array}\right]\left[\begin{array}{cc}1 / 2 & 1 / 2 \\ 1 / 2 & 3 / 2\end{array}\right]=$

$\left[\begin{array}{cc}-18 / 88 & -32 / 88 \\ 14 / 88 & 20 / 88\end{array}\right]$ take $\mathrm{B}=\left[\begin{array}{ll}a & b \\ c & d\end{array}\right]$ then $|B|=$

$\left[\begin{array}{ll}|a| & |b| \\ |c| & |d|\end{array}\right]$

$\mathrm{B}=\mathrm{D}_{\mathrm{y}}|B|+\mathrm{A}_{\mathrm{c}}^{-1} \Rightarrow\left[\begin{array}{ll}a & b \\ c & d\end{array}\right]=$ 
$\left[\begin{array}{cc}-18 / 88 & -32 / 88 \\ 14 / 88 & 20 / 88\end{array}\right]\left[\begin{array}{ll}|a| & |b| \\ |c| & |d|\end{array}\right]$

$+\left[\begin{array}{cc}-11 / 44 & 7 / 44 \\ 11 / 44 & -3 / 44\end{array}\right]$

By solving this we get,

$\mathrm{B}=\left[\begin{array}{cc}-0,2874 & 0.1494 \\ 0.2644 & -0.0575\end{array}\right]$

$(\mathbf{R}) \rightarrow($ B1 )

$\mathrm{y}=\left[\begin{array}{c}1 \\ -1\end{array}\right], \mathrm{A}_{\mathrm{ye}}=\left[\begin{array}{ll}1 & 3 \\ 6 & 7\end{array}\right] ; \mathrm{A}_{\mathrm{yf}}=\left[\begin{array}{ll}2 & 4 \\ 5 & 4\end{array}\right]$

$\mathrm{A}_{\mathrm{ye}}{ }^{-1} \mathrm{~A}_{\mathrm{yf}}=-\frac{1}{11}\left[\begin{array}{cc}7 & -3 \\ -6 & 1\end{array}\right]\left[\begin{array}{ll}2 & 4 \\ 5 & 4\end{array}\right]$

$=\left[\begin{array}{cc}1 / 11 & -16 / 11 \\ 7 / 11 & 20 / 11\end{array}\right]$

$\Delta_{1}=1 / 11 \geq 0$ and $\Delta_{2}=132 / 121 \geq 0$.

So $\mathrm{A}_{\mathrm{ye}}{ }^{-1} \mathrm{~A}_{\mathrm{yf}}$ is a P-Matrix

$(\mathbf{R}) \rightarrow(\mathrm{B2})$

take $\mathrm{y}=\left[\begin{array}{c}1 \\ -1\end{array}\right] ; \mathrm{A}_{\mathrm{ye}}{ }^{-1} \mathrm{~A}_{\mathrm{yf}}=\left[\begin{array}{cc}1 / 11 & -16 / 11 \\ 7 / 11 & 20 / 11\end{array}\right]$

$\mathrm{A}_{\mathrm{ye}}{ }^{-1} \mathrm{~A}_{\mathrm{yf}} \mathrm{x}>0 \Rightarrow\left[\begin{array}{cc}1 / 11 & -16 / 11 \\ 7 / 11 & 20 / 11\end{array}\right]\left[\begin{array}{l}x_{1} \\ x_{2}\end{array}\right]$

$>0 ; x=\left[\begin{array}{c}17 \\ 1\end{array}\right]>0$

$(\mathbf{R}) \rightarrow(\mathrm{B3})$

take $\mathrm{y}=\left[\begin{array}{c}1 \\ -1\end{array}\right] ; \mathrm{A}_{\mathrm{ye}}{ }^{-1}=-\frac{1}{11}\left[\begin{array}{cc}7 & -3 \\ -6 & 1\end{array}\right]$

$=\left[\begin{array}{cc}-7 / 11 & 3 / 11 \\ 6 / 11 & -1 / 11\end{array}\right]$

$\mathrm{A}_{\mathrm{ye}}{ }^{-1} \mathrm{x}=\left[\begin{array}{cc}-7 / 11 & 3 / 11 \\ 6 / 11 & -1 / 11\end{array}\right]\left[\begin{array}{l}x_{1} \\ x_{2}\end{array}\right]>0$; Solving this we get $\mathrm{x}=\left[\begin{array}{l}1 \\ 3\end{array}\right]$

$\mathrm{A}_{\mathrm{yf}}=\left[\begin{array}{ll}2 & 4 \\ 5 & 4\end{array}\right] ; \mathrm{A}_{\mathrm{yf}}{ }^{-1}=\left[\begin{array}{cc}-4 & 4 \\ 5 & -2\end{array}\right]$

$\mathrm{A}_{\mathrm{yf}}{ }^{-1} \mathrm{x}>0 \Rightarrow\left[\begin{array}{cc}-4 & 4 \\ 5 & -2\end{array}\right]\left[\begin{array}{l}x_{1} \\ x_{2}\end{array}\right]>0$; So the value of $\mathrm{x}$ is $\left[\begin{array}{l}1 \\ 2\end{array}\right]$

$(\mathbf{R}) \rightarrow\left(\begin{array}{l}\mathbf{B} 4 \\ )\end{array}\right)$ take $\mathrm{y}=\left[\begin{array}{c}1 \\ -1\end{array}\right]$; from previous examples we know $\mathrm{D}_{\mathrm{y}}=\left[\begin{array}{cc}-18 / 88 & -32 / 88 \\ 14 / 88 & 20 / 88\end{array}\right]$

$\mathrm{D}_{\mathrm{y}} \mathrm{x}=\left[\begin{array}{cc}-18 / 88 & -32 / 88 \\ 14 / 88 & 20 / 88\end{array}\right]\left[\begin{array}{l}x_{1} \\ x_{2}\end{array}\right]$

$=\left[\begin{array}{cc}-18 x_{1} / 88 & -32 x_{2} / 88 \\ 14 x_{1} / 88 & 20 x_{2} / 88\end{array}\right] ;\left|D_{\mathrm{y}} x\right|<\mathrm{x}$ means

$\left|-18 x_{1} / 88-32 x_{2} / 88\right|<\mathrm{x}_{1}$ and $\mid 14 x_{1} / 88$

$+20 x_{2} / 88 \mid<\mathrm{x}_{2}$

solving this we get $\mathrm{x}=\left[\begin{array}{l}1 \\ 1\end{array}\right]$

$(\mathbf{R}) \rightarrow(\mathrm{C1})$

$\mathrm{A}_{\mathrm{yz}}=\mathrm{A}_{\mathrm{c}}-\mathrm{T}_{\mathrm{y}} \Delta \mathrm{T}_{\mathrm{z}}$; take $\mathrm{y}=\left[\begin{array}{c}1 \\ -1\end{array}\right], \mathrm{z}=\left[\begin{array}{l}1 \\ 1\end{array}\right]$,

$$
\begin{aligned}
& \mathrm{y}^{\prime}=\left[\begin{array}{l}
-1 \\
-1
\end{array}\right], \mathrm{z}^{\prime}=\left[\begin{array}{c}
-1 \\
1
\end{array}\right] \\
& \mathrm{T}_{\mathrm{y}}=\left[\begin{array}{cc}
1 & 0 \\
0 & -1
\end{array}\right], \mathrm{T}_{\mathrm{z}}=\left[\begin{array}{cc}
1 & 0 \\
0 & 1
\end{array}\right], \mathrm{T}_{\mathrm{y}}{ }^{\prime}=\left[\begin{array}{cc}
-1 & 0 \\
0 & -1
\end{array}\right], \\
& \mathrm{T}_{\mathrm{z}}=\left[\begin{array}{cc}
-1 & 0 \\
0 & 1
\end{array}\right] ; \mathrm{A}_{\mathrm{c}}=\left[\begin{array}{cc}
3 / 2 & 7 / 2 \\
11 / 2 & 11 / 2
\end{array}\right] \\
& \mathrm{T}_{\mathrm{y}} \Delta \mathrm{T}_{\mathrm{z}}=\left[\begin{array}{cc}
1 & 0 \\
0 & -1
\end{array}\right]\left[\begin{array}{cc}
1 / 2 & 1 / 2 \\
1 / 2 & 3 / 2
\end{array}\right]\left[\begin{array}{cc}
1 & 0 \\
0 & 1
\end{array}\right]= \\
& {\left[\begin{array}{cc}
1 / 2 & 1 / 2 \\
-1 / 2 & -3 / 2
\end{array}\right]}
\end{aligned}
$$

$\mathrm{A}_{\mathrm{yz}}=\mathrm{A}_{\mathrm{c}}-\mathrm{T}_{\mathrm{y}} \Delta \mathrm{T}_{\mathrm{z}}=\left[\begin{array}{cc}3 / 2 & 7 / 2 \\ 11 / 2 & 11 / 2\end{array}\right]-$

$\left[\begin{array}{cc}1 / 2 & 1 / 2 \\ -1 / 2 & -3 / 2\end{array}\right]=\left[\begin{array}{ll}1 & 3 \\ 6 & 7\end{array}\right]$

$\mathrm{T}_{\mathrm{y}^{\prime}} \Delta \mathrm{T}_{\mathrm{z}}=\left[\begin{array}{cc}-1 & 0 \\ 0 & -1\end{array}\right]\left[\begin{array}{cc}1 / 2 & 1 / 2 \\ 1 / 2 & 3 / 2\end{array}\right]\left[\begin{array}{cc}-1 & 0 \\ 0 & 1\end{array}\right]=$

$\left[\begin{array}{ll}1 / 2 & -1 / 2 \\ 1 / 2 & -3 / 2\end{array}\right]$

$1 / 2 \quad-3 / 2]$

$\mathrm{A}_{\mathrm{y}^{\prime} \mathrm{z}^{\prime}}=\mathrm{A}_{\mathrm{c}}-\mathrm{T}_{\mathrm{y}^{\prime}} \Delta \mathrm{T}_{\mathrm{z}}{ }^{\prime}=\left[\begin{array}{cc}3 / 2 & 7 / 2 \\ 11 / 2 & 11 / 2\end{array}\right]-$

$\left[\begin{array}{ll}1 / 2 & -1 / 2 \\ 1 / 2 & -3 / 2\end{array}\right]=\left[\begin{array}{ll}1 & 4 \\ 5 & 7\end{array}\right]$

$\operatorname{det} \mathrm{A}_{\mathrm{yz}}=7-18=-11$

$\operatorname{det}{\mathrm{A}_{\mathrm{y}^{\prime}{ }^{\prime}{ }^{\prime}}}=7-20=-13$

$\left(\operatorname{det} \mathrm{A}_{\mathrm{yz}}\right)\left(\operatorname{det} \mathrm{A}_{\mathrm{y}^{\prime} \mathrm{z}^{\prime}}\right)=(-11) \cdot(-13)=143>0$

$(\mathbf{R}) \rightarrow(\mathrm{C2})$

take $\mathrm{y}=\left[\begin{array}{c}1 \\ -1\end{array}\right], \mathrm{y}^{\prime}=\left[\begin{array}{l}-1 \\ -1\end{array}\right], \mathrm{z}=\left[\begin{array}{l}1 \\ 1\end{array}\right] ; \mathrm{A}_{\mathrm{yz}}=\left[\begin{array}{cc}1 & 3 \\ 6 & 7\end{array}\right]$

$\mathrm{A}_{\mathrm{y}^{\prime} \mathrm{z}}=\mathrm{A}_{\mathrm{c}}-\mathrm{T}_{\mathrm{y}^{\prime}} \Delta \mathrm{T}_{\mathrm{z}}=\left[\begin{array}{cc}3 / 2 & 7 / 2 \\ 11 / 2 & 11 / 2\end{array}\right]-$

$\left[\begin{array}{cc}-1 & 0 \\ 0 & -1\end{array}\right]\left[\begin{array}{cc}1 / 2 & 1 / 2 \\ 1 / 2 & 3 / 2\end{array}\right]\left[\begin{array}{cc}1 & 0 \\ 0 & 1\end{array}\right]$

$\operatorname{det} \mathrm{A}_{\mathrm{yz}}=7-18=-11$

$$
=\left[\begin{array}{ll}
2 & 4 \\
6 & 7
\end{array}\right]
$$

$\operatorname{det} \mathrm{A}_{\mathrm{y}^{\prime} \mathrm{z}}=14-24=-10$

$\left(\operatorname{det} \mathrm{A}_{\mathrm{yz}}\right)\left(\operatorname{det} \mathrm{A}_{\mathrm{y}^{\prime}{ }_{\mathrm{z}}}\right)=(-11) \cdot(-10)=110>0$

$(\mathbf{R}) \rightarrow(\mathrm{C3})$

take $\mathrm{y}=\left[\begin{array}{c}1 \\ -1\end{array}\right]$ and $\mathrm{z}=\left[\begin{array}{l}1 \\ 1\end{array}\right] ; \mathrm{T}_{\mathrm{y}}=\left[\begin{array}{cc}1 & 0 \\ 0 & -1\end{array}\right], \mathrm{T}_{\mathrm{z}}$

$=\left[\begin{array}{ll}1 & 0 \\ 0 & 1\end{array}\right] ; \Delta=\left[\begin{array}{ll}1 / 2 & 1 / 2 \\ 1 / 2 & 3 / 2\end{array}\right]$

$\mathrm{T}_{\mathrm{y}} \Delta \mathrm{T}_{\mathrm{z}}=\left[\begin{array}{cc}1 / 2 & 1 / 2 \\ -1 / 2 & -3 / 2\end{array}\right] ; \mathrm{D}_{\mathrm{yz}}=\mathrm{A}_{\mathrm{c}}{ }^{-1} \mathrm{~T}_{\mathrm{y}} \Delta \mathrm{T}_{\mathrm{z}}$

$=\left[\begin{array}{cc}-11 / 44 & 7 / 44 \\ 11 / 44 & -3 / 44\end{array}\right]\left[\begin{array}{cc}1 / 2 & 1 / 2 \\ -1 / 2 & -3 / 2\end{array}\right]$

$=\left[\begin{array}{cc}-18 / 88 & -32 / 88 \\ 14 / 88 & 20 / 88\end{array}\right]$

So, after calculating the eigen value of $\mathrm{D}_{\mathrm{yz}}=0.117$ and -0.094 , where both are less than 1 .

$(\mathbf{R}) \rightarrow(\mathbf{C 4})$ 


$$
\begin{aligned}
& \text { take } \mathrm{y}=\left[\begin{array}{l}
-1 \\
-1
\end{array}\right] \text { and } \mathrm{z}=\left[\begin{array}{c}
-1 \\
1
\end{array}\right] \\
& \mathrm{T}_{\mathrm{y}}=\left[\begin{array}{cc}
-1 & 0 \\
0 & -1
\end{array}\right], \mathrm{T}_{\mathrm{z}}=\left[\begin{array}{cc}
-1 & 0 \\
0 & 1
\end{array}\right] \\
& \mathrm{A}_{\mathrm{c}}=\left[\begin{array}{cc}
3 / 2 & 7 / 2 \\
11 / 2 & 11 / 2
\end{array}\right] ; \Delta=\left[\begin{array}{cc}
1 / 2 & 1 / 2 \\
1 / 2 & 3 / 2
\end{array}\right] \\
& \mathrm{A}_{\mathrm{yz}}=\mathrm{A}_{\mathrm{c}}-\mathrm{T}_{\mathrm{y}} \Delta \mathrm{T}_{\mathrm{z}}=\left[\begin{array}{cc}
3 / 2 & 7 / 2 \\
11 / 2 & 11 / 2
\end{array}\right] \\
& \text { - }\left[\begin{array}{cc}
-1 & 0 \\
0 & -1
\end{array}\right]\left[\begin{array}{cc}
1 / 2 & 1 / 2 \\
1 / 2 & 3 / 2
\end{array}\right]\left[\begin{array}{cc}
-1 & 0 \\
0 & 1
\end{array}\right]=\left[\begin{array}{ll}
1 & 4 \\
5 & 7
\end{array}\right] \\
& \mathrm{A}_{\mathrm{yz}}{ }^{-1}=\left[\begin{array}{cc}
-7 / 13 & 4 / 13 \\
5 / 13 & -1 / 13
\end{array}\right] \\
& \mathrm{A}_{\mathrm{c}} \mathrm{A}_{\mathrm{yz}}{ }^{-1}=\left[\begin{array}{cc}
3 / 2 & 7 / 2 \\
11 / 2 & 11 / 2
\end{array}\right]\left[\begin{array}{cc}
-7 / 13 & 4 / 13 \\
5 / 13 & -1 / 13
\end{array}\right] \\
& =\left[\begin{array}{cc}
14 / 26 & 5 / 26 \\
-22 / 26 & 33 / 26
\end{array}\right] \\
& \left(\text { Ac } \mathrm{A}_{\mathrm{yz}}{ }^{-1}\right)_{11}=14 / 26=0.538>1 / 2 \\
& \left(\text { Ac } \mathrm{A}_{\mathrm{yz}}{ }^{-1}\right)_{22}=33 / 26=1.269>1 / 2 \\
& \text { ( } \mathbf{R}) \rightarrow(\text { C5 ) } \\
& \mathrm{A}_{\mathrm{c}}=\left[\begin{array}{cc}
3 / 2 & 7 / 2 \\
11 / 2 & 11 / 2
\end{array}\right] ; \mathrm{A}^{I}=\left[\begin{array}{ll}
{[1,2]} & {[3,4]} \\
{[5,6]} & {[4,7]}
\end{array}\right] \\
& \text { take } \mathrm{A}=\left[\begin{array}{ll}
1 & 3 \\
5 & 7
\end{array}\right] ; \mathrm{A}^{-1}=\left[\begin{array}{cc}
-7 / 8 & 3 / 8 \\
5 / 8 & -1 / 8
\end{array}\right] \\
& \mathrm{A}_{\mathrm{c}} \mathrm{A}^{-1}=\left[\begin{array}{cc}
3 / 2 & 7 / 2 \\
11 / 2 & 11 / 2
\end{array}\right]\left[\begin{array}{cc}
-7 / 8 & 3 / 8 \\
5 / 8 & -1 / 8
\end{array}\right] \\
& =\left[\begin{array}{cc}
14 / 16 & 2 / 16 \\
-22 / 16 & 22 / 16
\end{array}\right] \\
& \left(\mathrm{A}_{\mathrm{c}} \mathrm{A}^{-1}\right)_{11}=14 / 16>1 / 2 \\
& \left(\mathrm{~A}_{\mathrm{c}} \mathrm{A}^{-1}\right)_{22}=22 / 16>1 / 2
\end{aligned}
$$

\section{Algorithm for checking Sin- gularity of an Interval Ma- trix}

Definition 3.1. An intertval matrix $A^{I}$ is called singular interval matrix if it contains a singular matrix that is for any $\mathrm{A} \in \mathrm{A}^{I}$, det $\mathrm{A}=0$.

Example 3.1. $\operatorname{det} \mathrm{A}^{I}=\operatorname{det}\left[\begin{array}{ll}{[1,2]} & {[3,4]} \\ {[5,6]} & {[7,8]}\end{array}\right]=[-17$ $, 1]$

since $0 \in[-17,1]$; so $\mathrm{A}^{I}$ contains atleast one singular matrix.

\section{Algorithm: 6}

Step 0 :: Select a matrix A such that $\left|A-A_{\mathrm{c}}\right|$ $=\Delta$ [recommended: $\mathrm{A}_{\mathrm{ij}}=\underline{A}_{\mathrm{ij}}$ if $\left(\mathrm{A}_{\mathrm{c}}{ }^{-1}\right)_{\mathrm{ji}} \geq 0$ and $\mathrm{A}_{\mathrm{ij}}=\bar{A}_{\mathrm{ij}}$ otherwise]

Step 1:: Compute $\mathrm{A}^{-1}$.

Step $2:$ :If $K_{i}=\phi$ for each $i$, terminate the algorithm fails. where $K_{i}=\left\{j\right.$; such that $\left(A_{c^{-}}\right.$ A) $\left.)_{\mathrm{ij}} \mathrm{A}^{-1}{ }_{\mathrm{ji}}<0\right\}$.
Step 3:: Otherwise find $\mathrm{k}$ such that $\mathrm{K}_{\mathrm{k}} \neq \phi$ and $\psi_{\mathrm{k}}=\min \left\{\psi_{\mathrm{j}} ; \mathrm{K}_{\mathrm{j}} \neq \phi\right\}$ where

$$
\psi_{\mathrm{i}}=\sum_{j \in K_{\mathrm{i}}}\left(A_{\mathrm{c}}-A\right)_{\mathrm{ij}} A_{\mathrm{ji}}{ }^{-1}
$$

Step 4:: If $\psi_{\mathrm{k}} \leq-1 / 2$ terminate. $\mathrm{A}^{I}$ is singular.

Step 5:: Otherwise set $A_{k j}=\left(2 A_{c}-A\right)_{k j}$ for each $\mathrm{j} \in \mathrm{K}_{\mathrm{k}}$ and go to step 1 .

Example 3.2. We have taken an interval matrix $\mathrm{A}^{I}=\left[\begin{array}{ll}{[1,2]} & {[3,4]} \\ {[5,6]} & {[7,8]}\end{array}\right] ;$ Now we will verify this.

$$
\mathrm{A}_{\mathrm{c}}=\left[\begin{array}{cc}
3 / 2 & 7 / 2 \\
11 / 2 & 15 / 2
\end{array}\right]
$$

Step 0 : $\Delta=\left[\begin{array}{ll}1 / 2 & 1 / 2 \\ 1 / 2 & 1 / 2\end{array}\right] ; A=\left[\begin{array}{ll}2 & 4 \\ 6 & 8\end{array}\right]$

Since, $\left[\begin{array}{ll}2 & 4 \\ 6 & 8\end{array}\right]-\left[\begin{array}{cc}3 / 2 & 7 / 2 \\ 11 / 2 & 15 / 2\end{array}\right]=\left[\begin{array}{cc}1 / 2 & 1 / 2 \\ 1 / 2 & 1 / 2\end{array}\right]$;

$\left|A-A_{\mathrm{c}}\right|=\left[\begin{array}{ll}1 / 2 & 1 / 2 \\ 1 / 2 & 1 / 2\end{array}\right]=\Delta$

Step $1: A^{-1}=\left[\begin{array}{cc}-1 & 1 / 2 \\ 3 / 2 & -1 / 4\end{array}\right]$

Step 2: $A_{c}-A=\left[\begin{array}{cc}3 / 2 & 7 / 2 \\ 11 / 2 & 15 / 2\end{array}\right]-\left[\begin{array}{ll}2 & 4 \\ 6 & 8\end{array}\right]=$ $\left[\begin{array}{ll}-1 / 2 & -1 / 2 \\ -1 / 2 & -1 / 2\end{array}\right]$

$\left(\mathrm{A}_{\mathrm{c}}-\mathrm{A}\right)_{11} \mathrm{~A}_{11}{ }^{-1}=(-1 / 2)(-1)=1 / 2>0$ and $\left(\mathrm{A}_{\mathrm{c}}-\mathrm{A}\right)_{12} \quad \mathrm{~A}_{21}{ }^{-1}=(-1 / 2)(3 / 2)=-3 / 4<0$

$$
\mathrm{K}_{1}=2
$$

$\left(\mathrm{A}_{\mathrm{c}}-\mathrm{A}\right)_{21} \mathrm{~A}_{12}{ }^{-1}=(-1 / 2)(1 / 2)=-1 / 4 ; 0$ and $\left(\mathrm{A}_{\mathrm{c}}-\mathrm{A}\right)_{22} \quad \mathrm{~A}_{22}{ }^{-1}=(-1 / 2)(-1 / 4)=1 / 8>0$

$$
\mathrm{K}_{2}=1
$$

Step $3: \mathrm{K}_{1}=2 \neq \phi$ and $\mathrm{K}_{2}=1 \neq \phi$

$\psi_{1}=\sum_{j \in K_{1}}\left(A_{\mathrm{c}}-A\right)_{1 \mathrm{j}}$

$A_{\mathrm{j} 1}{ }^{-1}=\sum_{j=2}\left(A_{\mathrm{c}}-A\right)_{12} A_{21}{ }^{-1}=-3 / 4$

$\psi_{2}=\sum_{j \in K_{2}}\left(A_{\mathrm{c}}-A\right)_{2 \mathrm{j}} A_{\mathrm{j} 2}{ }^{-1}=\sum_{j=1}\left(A_{\mathrm{c}}-\right.$ A) ${ }_{21} A_{12}{ }^{-1}=-1 / 4$

$\psi_{\mathrm{k}}=\min \{-3 / 4,-1 / 4\}=-3 / 4=\psi_{1}$

Step $4: \psi_{1}=-3 / 4 \leq-1 / 2$

So according to the algorithm given $\mathrm{A}^{I}$ is singular.

\section{Theoretical Result on Sin- gular Interval Matrix}

Theorem 4.1. 7] An interval matrix $A^{I}$ is singular if and only if it satisfies $\left|A_{c} x\right| \leq \Delta|x|$; for some non zero vector $x$

Example 4.1. take $\mathrm{A}^{I}=\left[\begin{array}{ll}{[1,2]} & {[3,4]} \\ {[5,6]} & {[7,8]}\end{array}\right]$;

$A_{c}=\left[\begin{array}{cc}3 / 2 & 7 / 2 \\ 11 / 2 & 15 / 2\end{array}\right] ; \Delta=\left[\begin{array}{cc}1 / 2 & 1 / 2 \\ 1 / 2 & 1 / 2\end{array}\right]$ 


$$
\begin{aligned}
& \left|A_{\mathrm{c}} x\right| \leq \Delta|x| \Rightarrow\left|\left[\begin{array}{cc}
3 / 2 & 7 / 2 \\
11 / 2 & 15 / 2
\end{array}\right]\left[\begin{array}{l}
x_{1} \\
x_{2}
\end{array}\right]\right| \\
\leq & {\left[\begin{array}{ll}
1 / 2 & 1 / 2 \\
1 / 2 & 1 / 2
\end{array}\right]\left|\left[\begin{array}{l}
x_{1} \\
x_{2}
\end{array}\right]\right| }
\end{aligned}
$$

solving this we get $\mathrm{x}=\left[\begin{array}{c}-3 \\ 2\end{array}\right]$

Conversely, take $A_{c}=\left[\begin{array}{cc}3 / 2 & 7 / 2 \\ 11 / 2 & 15 / 2\end{array}\right]$;

$\Delta=\left[\begin{array}{ll}1 / 2 & 1 / 2 \\ 1 / 2 & 1 / 2\end{array}\right]$ and $\mathrm{x}=\left[\begin{array}{c}-3 \\ 2\end{array}\right]$ which satisfies the above inequality.

So, from $\mathrm{A}_{\mathrm{c}}$ and $\Delta$ we can get $\mathrm{A}^{I}=\left[\mathrm{A}_{\mathrm{c}}-\Delta\right.$, $\left.\mathrm{A}_{\mathrm{c}}+\Delta\right]=\left[\begin{array}{ll}{[1,2]} & {[3,4]} \\ {[5,6]} & {[7,8]}\end{array}\right]$, which is a singular matrix.

\section{Eigen Values of an Interval Matrix}

Definition 5.1. Let $A \in M_{n x n}(R)$. A nonzero vector $\mathrm{v} \in \mathrm{R}^{n}$ is called an eigen vector of $\mathrm{A}$ if $\mathrm{Av}=$ $\lambda \mathrm{v}$ for some scalar $\lambda$. The scalar $\lambda$ is called the eigenvalue of $\mathrm{A}$.

Example 5.1. Let $A=\left[\begin{array}{ll}1 & 1 \\ 4 & 1\end{array}\right]$; we will get eigenvalues and eigen vectors after solving $\mathrm{Av}=\lambda \mathrm{v}$.

$\left[\begin{array}{ll}1 & 1 \\ 4 & 1\end{array}\right] \mathrm{v}=\lambda \mathrm{v}$; solving this we get eigen values are $3,-1$ and corrosponding eigen vectors are $\left[\begin{array}{l}1 \\ 2\end{array}\right]$ and $\left[\begin{array}{c}1 \\ -2\end{array}\right]$ respectively.

Theorem 5.1. 7] $\lambda \in \mathrm{L}$ if and only if the interval matrix $\left[\left(\mathrm{A}_{\mathrm{c}}-\lambda \mathrm{I}\right)-\Delta,\left(\mathrm{A}_{\mathrm{c}}-\lambda \mathrm{I}\right)+\Delta\right]$ is singular matrix. where $L=\left\{\lambda \in R ; A x=\lambda x\right.$ for some, $A \in A^{I}, x$ $\neq 0\}$

Example 5.2. Take $\mathrm{A}^{I}=\left[\begin{array}{ll}{[1,3]} & {[3,5]} \\ {[5,7]} & {[7,9]}\end{array}\right] ; \mathrm{A}_{\mathrm{c}}=$ $\left[\begin{array}{ll}2 & 4 \\ 6 & 8\end{array}\right] ; \Delta=\left[\begin{array}{ll}1 & 1 \\ 1 & 1\end{array}\right]$

Take any $\mathrm{A} \in \mathrm{A}^{I} ; \mathrm{A}=\left[\begin{array}{ll}3 & 4 \\ 7 & 9\end{array}\right]$; say eigen values of $\mathrm{A}$ are $\lambda_{1}$ and $\lambda_{2}$

$\lambda_{1}=12.0827$ and $\lambda_{2}=-0.08276$

$\left(\mathrm{A}_{\mathrm{c}}-\lambda_{1} \mathrm{I}\right)-\Delta=\left(\mathrm{A}_{\mathrm{c}}-\lambda_{1} \mathrm{I}\right)+\Delta=\left(\left[\begin{array}{ll}2 & 4 \\ 6 & 8\end{array}\right]\right.$ -

$\left.\left[\begin{array}{cc}12.082 & 0 \\ 0 & 12.082\end{array}\right]\right)+\left[\begin{array}{ll}1 & 1 \\ 1 & 1\end{array}\right]=\left[\begin{array}{cc}-9.082 & 5 \\ 7 & -3.082\end{array}\right]$

Now the new interval matrix will be $=\left[\left(\mathrm{A}_{\mathrm{c}}-\right.\right.$ $\left.\left.\lambda_{1} \mathrm{I}\right)-\Delta,\left(\mathrm{A}_{\mathrm{c}}-\lambda_{1} \mathrm{I}\right)+\Delta\right]$

$$
\mathrm{A}^{I}=\left[\begin{array}{cc}
{[-11.082,-9.082]} & {[3,5]} \\
{[5,7]} & {[-5.082,-3.082]}
\end{array}\right]
$$

$\mathrm{A}_{\mathrm{c}}{ }^{\prime}=\left[\begin{array}{cc}-10.082 & 4 \\ 6 & -4.082\end{array}\right]$ and $\Delta^{\prime}=\left[\begin{array}{ll}1 & 1 \\ 1 & 1\end{array}\right]$
Since we know $\mathrm{A}^{I}$ is singular if and only if it satisfies $\left|A_{\mathrm{c}} x\right| \leq \Delta|x|$; for some non zero vector $\mathrm{x}$

$$
\begin{aligned}
& \left|A_{\mathrm{c}}{ }^{\prime} x\right| \leq \Delta^{\prime}|x| \Rightarrow\left|\left[\begin{array}{cc}
-10.082 & 4 \\
6 & -4.082
\end{array}\right]\left[\begin{array}{l}
x_{1} \\
x_{2}
\end{array}\right]\right| \\
\leq & {\left[\begin{array}{ll}
1 & 1 \\
1 & 1
\end{array}\right]\left|\left[\begin{array}{l}
x_{1} \\
x_{2}
\end{array}\right]\right| }
\end{aligned}
$$

Solving this we get $\mathrm{x}=\left[\begin{array}{l}1 \\ 2\end{array}\right]$; So $\mathrm{A}^{I^{\prime}}$ is singular.

Conversely, take $A_{c}=\left[\begin{array}{ll}2 & 4 \\ 6 & 8\end{array}\right], \Delta=\left[\begin{array}{ll}1 & 1 \\ 1 & 1\end{array}\right]$ and $\lambda=12.0827$

Then $\left[\left(\mathrm{A}_{\mathrm{c}}-\lambda \mathrm{I}\right)-\Delta,\left(\mathrm{A}_{\mathrm{c}}-\lambda \mathrm{I}\right)+\Delta\right]=$ $\left[\begin{array}{cc}{[-11.082,-9.082]} & {[3,5]} \\ {[5,7]} & {[-5.082,-3.082]}\end{array}\right]$ is singular.

for $\lambda=12.0827 \exists \mathrm{A}=\left[\begin{array}{ll}3 & 4 \\ 7 & 9\end{array}\right] \in\left[\begin{array}{ll}{[1,3]} & {[3,5]} \\ {[5,7]} & {[7,9]}\end{array}\right]$

Theorem 5.2. 7] Let $\lambda \in L$, then $\exists x \neq 0, p \neq 0$ and $y, z \in Y$ and $d \in[0,1]$ such that,

1) $\left(A_{c}-d T_{y} \Delta T_{z}\right) x=\lambda x$

2) $\left(A_{c}-d T_{y} \Delta T_{z}\right)^{t} p=\lambda p$

3) $T_{z} x \geq 0$

4) $T_{z} p \geq 0$

Note : When $\mathrm{A}^{I}$ is a $2 \times 2$ interval matrix then

$$
Y=\left\{\left[\begin{array}{l}
1 \\
1
\end{array}\right],\left[\begin{array}{c}
1 \\
-1
\end{array}\right],\left[\begin{array}{c}
-1 \\
1
\end{array}\right],\left[\begin{array}{l}
-1 \\
-1
\end{array}\right]\right\}
$$




\section{Present Contribution for de- termining Eigen Value}

\subsection{Matlab Code for 2x2 matrix}

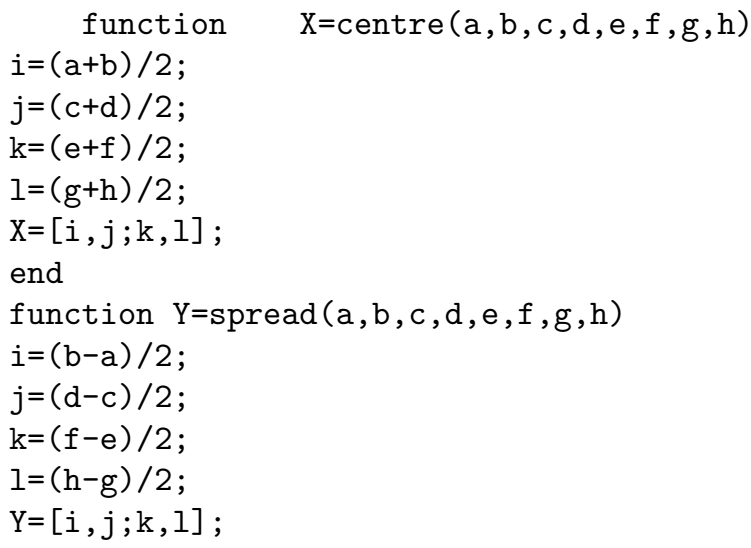

Table 1

\begin{tabular}{|c|c|c|c|c|c|}
\hline Matrix & $\mathrm{y}$ & $\mathrm{Z}$ & $\begin{array}{l}\text { Eigen } \\
\text { Value }\end{array}$ & $\begin{array}{l}\text { Eigen } \\
\text { Vectors } \\
(\mathrm{x} \text { and } \\
\mathrm{p})\end{array}$ & d \\
\hline$\left[\begin{array}{ll}{[1,3]} & {[4,6]} \\
{[6,8]} & {[6,10]}\end{array}\right]$ & {$\left[\begin{array}{c}1 \\
-1\end{array}\right]$} & {$\left[\begin{array}{c}-1 \\
1\end{array}\right]$} & $\begin{array}{l}- \\
1.6311\end{array}$ & $\begin{array}{l}{\left[\begin{array}{c}-0.8090 \\
0.5878\end{array}\right]} \\
; \\
{\left[\begin{array}{c}-0.8876 \\
0.4606\end{array}\right]}\end{array}$ & 0.001 \\
\hline$\left[\begin{array}{cc}{[1,3]} & {[5,7]} \\
{[9,11]} & {[14,15]}\end{array}\right]$ & {$\left[\begin{array}{c}1 \\
-1\end{array}\right]$} & {$\left[\begin{array}{c}-1 \\
1\end{array}\right]$} & $\begin{array}{l}- \\
1.7013\end{array}$ & $\begin{array}{l}{\left[\begin{array}{c}-0.8510 \\
0.5252\end{array}\right]} \\
; \\
{\left[\begin{array}{c}-0.9378 \\
0.3472\end{array}\right]}\end{array}$ & 0.001 \\
\hline$\left[\begin{array}{ll}{[10,12]} & {[14,16]} \\
{[18,20]} & {[22,24]}\end{array}\right]$ & {$\left[\begin{array}{c}1 \\
-1\end{array}\right]$} & {$\left[\begin{array}{c}-1 \\
1\end{array}\right]$} & $\begin{array}{l}- \\
0.9145\end{array}$ & $\begin{array}{l}{\left[\begin{array}{c}-0.7830 \\
0.6220\end{array}\right]} \\
; \\
{\left[\begin{array}{c}-0.8472 \\
0.5313\end{array}\right]}\end{array}$ & 0.001 \\
\hline
\end{tabular}

\section{Conclusion}

In this paper, we have calculated the eigen value of an interval matrix using singularity property and developed matlab code.

Acknowledgement : I am heartily thankful to Prof. Geetanjali Panda, IIT Kharagpur for her constant encouragement throughout this work. Her valuable advices and guidance were a constant source of inspiration for me.

\section{References}

[1] Paul S Dwyer. "Linear computations." In: (1951).

[2] Teruo Sunaga. "Theory of interval algebra and its application to numerical analysis". In: RAAG memoirs 2.29-46 (1958), p. 209.

[3] Ramon E Moore. Interval analysis. Vol. 4. Prentice-Hall Englewood Cliffs, NJ, 1966.

[4] Jiřı Rohn. "Interval linear systems with prescribed column sums". In: Linear algebra and its applications 39 (1981), pp. 143-148.

[5] Jiřı Rohn. "Solving systems of linear interval equations". In: Reliability in Computing. Elsevier, 1988, pp. 171-182.

[6] Jiri Rohn. "Systems of linear interval equations". In: Linear algebra and its applications 126 (1989), pp. 39-78.

[7] Jiri Rohn. "Interval matrices: Singularity and real eigenvalues". In: SIAM journal on matrix analysis and applications 14.1 (1993), pp. 8291.

[8] Jirı Rohn. "Inverse interval matrix". In: SIAM Journal on Numerical Analysis 30.3 (1993), pp. $864-870$.

[9] Ramon E Moore, $\mathrm{R}$ Baker Kearfott, and Michael J Cloud. Introduction to interval analysis. Vol. 110. Siam, 2009. 